\title{
Aplicación del algoritmo de solución paso-a-paso de la ecuación que determina la estabilidad de un generador síncrono
}

\author{
H.A. Grajales-Román \\ División de Ingeniería Eléctrica y Departamento de Ingeniería Eléctrica de Potencia \\ Facultad de Ingeniería, UNAM \\ E-mail: alfhugo@gmail.com
}

(Recibido: marzo de 2006; aceptado: septiembre de 2006)

\section{Resumen}

La solución de la ecuación diferencial de orden dos, que representa en forma matemámática la dinámica de máquinas generadoras de corriente alterna, requiere de un algoritmo para su solución. La traducción a lenguaje de computadora del método paso-a-paso para la solución de la ecuación de oscilación que representa la dinámica del generador, permite el análisis de redes eléctricas cuando son sometidas a cambios repentinos que provocan oscilaciones de frecuencia, y por lo tanto, de tensión. El algoritmo se basa en la consideración del hecho que; se puede calcular un nuevo valor del ángulo $\delta$, si se conoce su valor de cambio en el intervalo anterior y se conoce la potencia acelerante en el nuevo intervalo de estudio. Con el apoyo del programa de computo llamado Matlab, se pueden realizar un sin número de corridas con valores diferentes de los parámetros del sistema, así como de los tiempos de apertura de los interruptores. Como resultado, el programa despliega la tendencia del rotor conocida como curva de oscilación.

Descriptores: Ecuación diferencial, integración, lenguaje de computadora.

\begin{abstract}
Thesecondorderdifferen tialequation solution, which mathematicallyrepresents the dy namic of altern cur rent gen er at ing ma chines, re quires an al go rithm for its so lu tion. The com puter ma chine lan guage trans la tion of step-by-step method for thesolu tion of swing ingequation which represents the dy namicgen er a tor, allows the anal y sis of elec trical net works when are sub ject to sud den changes that mo tive os cil la tion of fre quency and there fore ten sion. The al go rithm is based on the fact of the pos si bil ity to ob tain a new value of the ä (delta) an gle, as long as its value is known in the preced ing in ter val and its ac cel er at ing power is known in the new study in terval. With the sup port of Mathlab soft ware, it is pos sible to ac com plish end less number of runs with many differ ent val ues and sys tem pa rameters, as well as time open ing of cir cuit break ers. As a re sult, the pro gram plots a curve that shows the os cil la tion ten dency.
\end{abstract}

Keywords:Differen tialequation, integral, computation language. 


\section{Introducción}

Los sistemas eléctricos de potencia están sujetos a cambios de cargas, algunas graduales y otras bruscas, unas por conexión de cargas ligeras y otras como fallas en las líneas de conducción, o en sus torres soportes, causando por lo tanto, que la estabilidad del sistema de transmisión de energía eléctrica entre en crisis.

Se define como límite de estabilidad en estado permanente de un generador o de un sistema, a la máxima potencia que puede ser transmitida a cambios de carga que permitan ajustes de excitación suficientes como para que se recupere el valor normal de la tensión que se tenía antes del cambio.

Si el ajuste de excitación se presenta con o inmediatamente después del cambio de carga, el limite de estabilidad en estas condiciones es llamado "límite dinámico del estado permante".

La bondad, flexibilidad o rigidez de un sistema eléctrico de potencia, dependerá de los elementos que lo conforman para este fin, como son, dispositivos de control, regula- ción, excitación, protección, elevación y reducción, por mencionarlos; de los cuales, algunos permanecen censando, otros enviando información sobre el estado y otros operando para mantener el sistema dentro de los parámetros de seguridad.

\section{La base de los sistemas, el generador}

De estos sistemas, el más importante por su razón de ser, es el generador de corriente alterna, también conocido como generador síncrono.

La expresión matemática de una fase del generador síncrono de polos lisos conforme a la ley de tensiones de Kirchhoff es:

$$
\bar{E}_{A C}=\bar{V}+\left(r_{A}+j X_{d}\right) \bar{I}_{A}[\mathrm{v}]
$$

y su representación fasorial se muestra en la figura 1.

En donde:

$E_{A C}:$ tensión inducida en las bobinas de la armadura

V: tensión en las terminales del generador

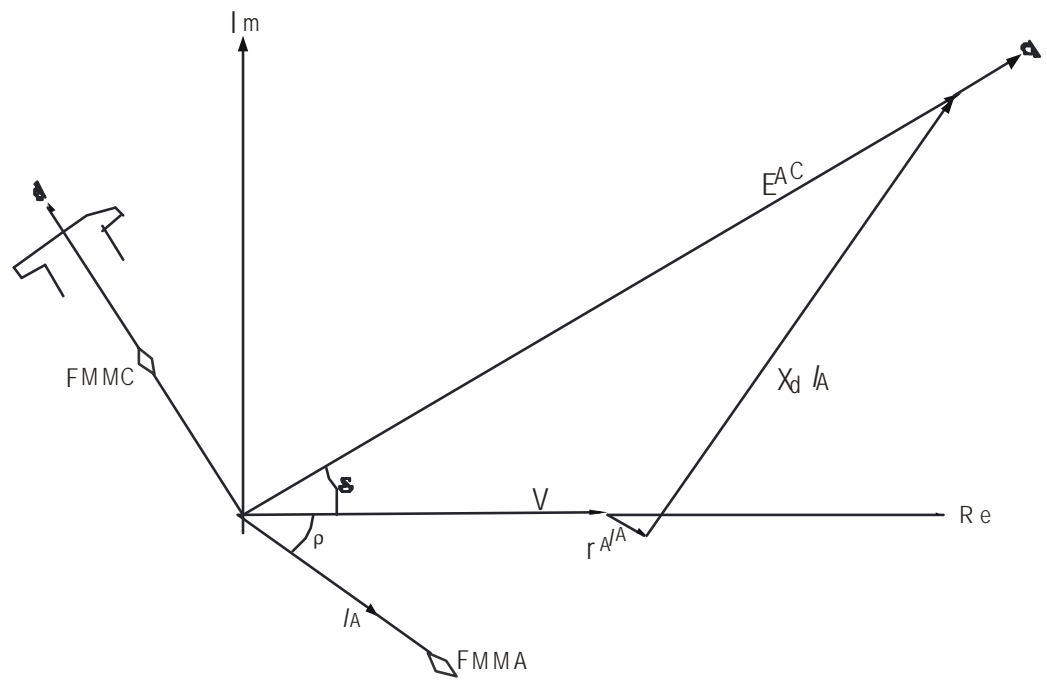

Figura 1. Representación fasorial de un generador síncrono de polos lisos 
$I_{A}$ : corriente de armadura

$r_{A} y X_{d}: r_{A}$ : valor de la resistencia de los conductores que conforman las bobinas de la armadura y $X_{d}$ : el valor de la característica de magnetización que genera la corriente de armadura en las laminaciones de acero en donde están alojadas las bobinas.

Esta representación del generador es por fase, siendo los generadores trifásicos.

El resultado de la expresión (1), $E_{A C}$, es un vector que tiene una dirección determinada por el ángulo $\delta$. Este ángulo representa en grados eléctricos la desviación que existe entre la tensión inducida en las bobinas de la armadura y la tensión en las terminales de la máquina.

Por otro lado, del desarrollo de la potencia aparente se determina la potencia real o activa que el generador entrega en sus terminales al sistema eléctrico y que resulta ser

$$
P=\frac{E_{A C}{ }^{*} V}{X_{d}} \operatorname{sen} \delta[w / \text { fase }]
$$

La potencia trifásica es la expresión (2) multiplicada por 3. Como lo muestra la expresión (2), la potencia que entrega un generador es directamente proporcional al producto de la tensión inducida en las bobinas de la armadura por la tensión en sus terminales e inversamente proporcional al valor de la reactancia que separa estas dos tensiones. La representación de la expresión (2) es una senoide como se muestra en la figura 2, y el valor de generación de potencia máxima se presenta precisamente cuando el ángulo entre la tensión inducida y la tensión en el extremo final de la reactancia que las separa, que es la de las terminales, tiene un valor de $90^{\circ}$.

Cuando $\mathrm{d}=90^{\circ}$

$$
P_{M A X}=\frac{E_{A C}^{*} V}{X_{d}}[w]
$$

El control que se tiene sobre los generadores conectados a un sistema o sistemas es entre otros, el de la velocidad de rotación. Si un generador aumenta o disminuye su velocidad, lo mismo lo hace la frecuencia de generación. La frecuencia en México es de 60 Hertz y se mantiene en toda la red eléctrica.

$$
f=\frac{P}{2} \times \frac{n}{60}[\text { Hert } z]
$$

En donde $f$ es la frecuencia, $P$ el número de polos del rotor y $n$ las revoluciones por minuto del rotor. Como se mencionó anteriormente, la ocurrencia de algún tipo de falla, hace que todo el sistema interconectado actúe de forma que la sección que circunda la

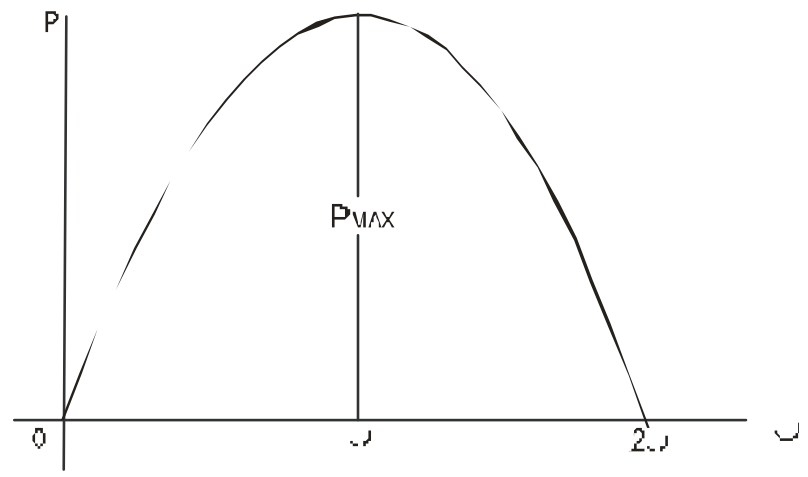

Figura 2. Curva Ángulo-Potencia de un generador síncrono de polos lisos 
Aplicación del algoritmo de solución paso-a-paso de la ecuación que determina la estabilidad ...

falla pueda ser aislada eléctricamente. Lo anterior es de gran trascendencia, puesto que equivale afirmar que en el tiempo más corto posible después de ocurrida una falla en algún punto de la red de suministro, el sistema continúe activo, logrando equilibrarse con las máquinas generadoras que continúan activas y aportando la alimentación eléctrica lo más estable posible.

\section{Leyes de la dinámica del rotor}

Por la relación existente entre la frecuencia y las revoluciones de giro del rotor, continuamente se hacen ejercicios y pruebas a ciertas partes de los sistemas eléctricos. El más riguroso es precisamente la simulación de la estabilidad de los generadores interconectados. Con el apoyo de programas de computo se simula el comportamiento dinámico de los generadores, ya sea individualmente o interconectados, y se analizan sus resultados para posibles prevenciones.

La transformación de la energía se realiza a través del rotor del generador. La máquina primaria aporta su potencia al rotor del generador y éste, en su rotación alrededor de las bobinas del estator, induce una tensión eléctrica que debe ser igual en unidades de potencia a la aplicada al rotor. La corriente que demanda la carga es suministrada por el valor de la excitación de los polos del rotor y de la potencia de la máquina impulsora. Las tres corrientes monofásicas generan en el núcleo del estator un campo magnético giratorio.

Al presentarse una falla en el sistema, la potencia eléctrica que aporta el generador sufre un cambio en el campo magnético giratorio y éste como se separa del eje del rotor, acciona a favor o en contra del par aplicado por la máquina impulsora, dependiendo de la característica de la falla.
Durante el corto tiempo que dura la respuesta de control sobre el rotor, éste pierde sincronismo y se va separando del eje magnético del campo giratorio.

La diferencia de potencia entre la entrada y la salida presenta un desequilibrio. Esta diferencia es, naturalmente, una potencia acelerante o desacelerante, según sea la magnitud de la potencia de salida. Así

$$
P_{a}=P_{m e c}-P_{\text {eléc }}[w]
$$

Cuando la potencia eléctrica es mayor a la mecánica, la potencia eléctrica está frenando a la máquina impulsora, por lo tanto, es una potencia desacelerante. Lo contrario a lo anterior, es una potencia acelerante.

La ecuación matemática que resuelve la expresión 3, se deriva de los principios de la cinemática. Sabemos que el trabajo es igual a fuerza por distancia o en fórmula para trabajo circular.

$$
T=F \times r \times \theta \quad[K g-m-r a d]
$$

La diferencial en el tiempo del trabajo es

$$
\left.d(T)=\tau \frac{d \theta}{d t}=\tau \omega \quad \text { [Joules }\right]
$$

? es el desplazamiento circular del rotor.

Por otro lado, la energía cinética de un cilindro macizo es

$$
E C=\frac{1}{2} \frac{m \times r^{2}}{g} \omega^{2} \quad \text { [Joules] }
$$

En donde $m$ es la masa del cilindro en $\mathrm{Kg}$. y $r$ su radio en metros.

Cuando la velocidad angular aumenta en $\mathrm{d} \omega$, el incremento de energ ía cinética es 


\section{H.A. Grajales-Román}

$$
d(E C)=\frac{m \times r^{2}}{g} \omega \frac{d \omega}{d t} \quad \text { [Joules] }
$$

Puesto que el trabajo realizado es igual al incremento de energía cinética, podemos escribir

$$
\begin{gathered}
d(T)=d(E C) \\
\tau \frac{d \theta}{d t}=\frac{m \times r^{2}}{g} \omega \frac{d \omega}{d t}
\end{gathered}
$$

$\mathrm{y}$

$$
\tau=I \omega \frac{d \omega}{d \theta}=I \alpha \quad[\text { Newtons }-m]
$$

En donde

$$
I=\frac{m \times r^{2}}{g}
$$

momento de inercia de la masa $m\left[\mathrm{Kg}-\mathrm{m}^{2}\right]$

$\alpha=\omega \frac{d \theta}{d \omega}$ aceleración angular $\left[\mathrm{rad}-\mathrm{seg}^{2}\right]$

$M=I \omega m o m e n t o$ angular[ Joules $-\mathrm{seg} / \mathrm{rad}]$

Multiplicando la expresión (5) por la velocidad angular?, tendremos la potencia.

Entonces:

$$
\begin{gathered}
\tau \omega=P a=I \omega \alpha=M \alpha \quad[\text { watts }] \\
P a=M \alpha=M \frac{d^{2} \theta}{d t^{2}} \quad[\text { watts }]
\end{gathered}
$$

(6) representa el valor de la expresión (3), por lo que reescribiendo

$$
P a=M \alpha=P_{m \mathfrak{c}}-P_{\text {eléc }} \quad[\text { watts }]
$$

$\theta$ es en grados geométricos, la posición que guarda el polo al estar girando con respecto a una referencia en el estator. Estos grados irán en aumento a manera que transcurre el tiempo. Sin embargo, la corriente de armadura genera un campo magnético que gira en el estator y es estacionario con relación al rotor; sin embargo, siguen teniendo distintas referencias.

Idealmente instalaremos una tercera referencia en la que las otras dos coincidan (Figura 3).

$$
\theta=\omega s t+\delta \quad[\text { grados }]
$$

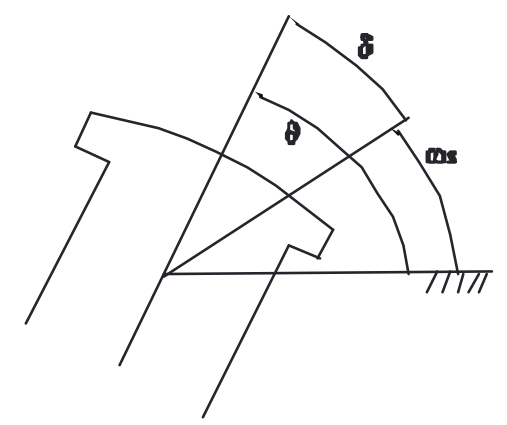

Figura 3. Determinación del ángulo $\delta$

Derivando dos veces

$$
\frac{d \theta}{d t}=\omega_{s}+\frac{d \delta}{d t}
$$

$$
\frac{d^{2} \theta}{d t^{2}}=\frac{d^{2} \delta}{d t^{2}}=\alpha
$$

Entonces podemos escribir (7)

$$
M \frac{d^{2} \delta}{d t^{2}}=P_{a}=P_{m e c}-P_{d e c} \quad[\text { watts }]
$$

Que es la ecuación diferencial que representa al generador síncrono para un estudio de estabilidad.

Para diferenciarlos entre varios generadores, al momento angular o constante de 
inercia $M$, hay que definirla con las características individuales de los generadores.

$$
\begin{gathered}
H=\frac{\text { energía almacenadaa vel.sin crona }}{\text { poten cia nominal }} \\
H=\frac{\text { Energía cinética }}{S_{N}}=\frac{\frac{1}{2} I \omega^{2}}{S_{N}}
\end{gathered}
$$

como

$$
S_{N} H=\frac{1}{2} M \omega_{s}
$$

momento cinético en dondews es la velocidad síncrona, despejando

$$
M=\frac{2 H}{\omega_{S}} S_{N} \quad\left[\frac{\text { mega Joules }}{\text { rads.mec }}\right]
$$

Sustituyendo en (8)

$$
\frac{2 H}{\omega_{S}} \frac{d^{2} \delta}{d t^{2}}=\frac{P_{a}}{S_{N}}=\frac{1}{S_{N}}\left(P_{m e c}-P_{e l e c}\right) \quad[p u]
$$

finalmente si

$$
\omega_{S}=2 \pi f=360^{\circ} f \quad\left[{ }^{\circ} \text { Eléc }\right]
$$

$\mathrm{y}$

$$
M=\frac{2 H}{\omega_{s}} \text { en }\left[\frac{\text { mega Joules }- \text { seg }}{{ }^{\circ} \text { Eléctricos }}\right]
$$

entonces

$$
\frac{H}{180 f} \frac{d^{2} \delta}{d t^{2}}=P_{m e c}-P_{\text {eléc }} \quad[w a t t s]
$$

El presente trabajo muestra la solución de la ecuación de oscilación de un generador síncrono en un sistema de dos líneas co- nectadas en paralelo, a una carga que demanda la energía que envía el generador, como se muestra en la figura 5. Apoyándose en el programa Matlab, se muestra como resultado la trayectoria del ángulo $\delta$ a la aplicación de una falla sostenida. Traducido a lenguaje de computadora el algoritmo de solución paso-a-paso, se pueden realizar numerosos ensayos en donde se pueden cambiar los valores de los parámetros involucrados como la potencia que se transfiere en el momento de la falla, características del generador, características de las líneas y transformadores, tiempos de apertura de los interruptores que limpian la falla, parámetros de la carga, etc.

En el estudio de la dinámica del rotor, la potencia mecánica es considerada constante, puesto que es la potencia real aplicada al rotor proveniente de la máquina impulsora y que éste transforma en energía eléctrica por medio de la excitación principal y el campo magnético giratorio. Durante el tiempo de respuesta del sistema de control de la máquina impulsora, el rotor del generador se desliza a valores peligrosos, y es aquí donde entran en juego las características del sistema eléctrico en su totalidad.

Una disminución en el par electromagnético, el par acelerante aumenta. En el tiempo que tardan los mecanismos de control para ajustar a la velocidad síncrona, el rotor se desliza de su posición hacia valores mayores y alcanzará valores que ponen en crisis la estabilidad del sistema eléctrico. La solución de la ecuación dinámica del generador, consiste en calcular el ángulo $\delta$ en función del tiempo, durante un período suficiente para determinar si $\delta$ crecerá sin límite o alcanza un máximo y tiende a regresar. 


\section{Análisis del método}

En el método de integración paso-a-paso para resolver la ecuación diferencial, que es un método entre varios existentes, se declara el más práctico y de buena exactitud, el cual manifiesta las siguientes suposiciones:

1. La potencia acelerante $P_{a}$ calculada al principio de un intervalo es constante desde la mitad del intervalo anterior hasta la mitad del intervalo en estudio.

2. La velocidad angular $\omega$, es constante durante cualquier intervalo calculado a la mitad del intervalo.

Naturalmente, estas suposiciones no se cumplen, puesto que $\delta$ cambia continuamente, y por lo tanto, también lo hacen $P_{a} \mathrm{y}$ ?. Si embargo, si los tiempos son lo suficientemente pequeños, estas consideraciones son bastante aceptables (Figura 4).

La potencia acelerante es calculada para los puntos 3, 2 y 1 que son los fines de los intervalos $n-1, n, n+1$. La curva de $P_{a}$, representa la suposición de que $\mathrm{P}_{a}$ es constante en puntos medios de los intervalos (Figura 4a).

De manera semejante, ? que representa el exceso de velocidad síncrona ?s, se muestra como un escalón que es constante durante el intervalo con valor determinado a la mitad del mismo. Entre las ordenadas $n-3 / 2$ y $n-1 / 2$ existe un cambio de velocidad causada por el valor constante de $P_{a}$ (Figura $4 b$ ).

El cambio de velocidad es igual al producto de la potencia acelerante por el valor del intervalo. Así

$$
\omega_{n-1 / 2}^{\prime}-\omega_{n-3 / 2}^{\prime}=\frac{d^{2} \delta}{d t^{2}} \Delta t=\frac{P_{a(n-1)}}{M} \Delta t
$$

El cambio de $\delta$ en cualquier intervalo, es el producto de $\omega$ por el intervalo y el tiempo de duración del mismo.

El cambio de $\delta$ durante el intervalo $n-1$ (Figura 4c) es

$$
\begin{gathered}
\Delta \delta n-1=\Delta \delta n-1-\Delta \delta n-2=\omega s_{n-3 / 2} \Delta t \\
\Delta \delta n=\Delta \delta n-1+\frac{P a_{(n-1)}}{M}(\Delta t)^{2}
\end{gathered}
$$

La expresión (8) calcula el cambio de d durante un intervalo si se conoce su valor en el intervalo anterior, y la potencia acelerante en el intervalo precedente. Así, la potencia acelerante debe calcularse al principio de cada intervalo para obtener suficientes puntos de la curva de oscilación.

Estas instrucciones se repiten durante el proceso de cálculo acomodando la instrucción correspondiente, según sea el tiempo de abertura de dos interruptores a la vez o de dos con diferentes tiempos de operación.

El programa anexo, desarrolla los cálculos y presenta la respuesta en forma gráfica de un generador síncrono aportando su potencia a una carga conectada al final de dos líneas paralelas, como se muestra la figura 5. 
DOI: http://dx.doi.org/10.22201/fi.25940732e.2007.08n3.012

Aplicación del algoritmo de solución paso-a-paso de la ecuación que determina la estabilidad ...

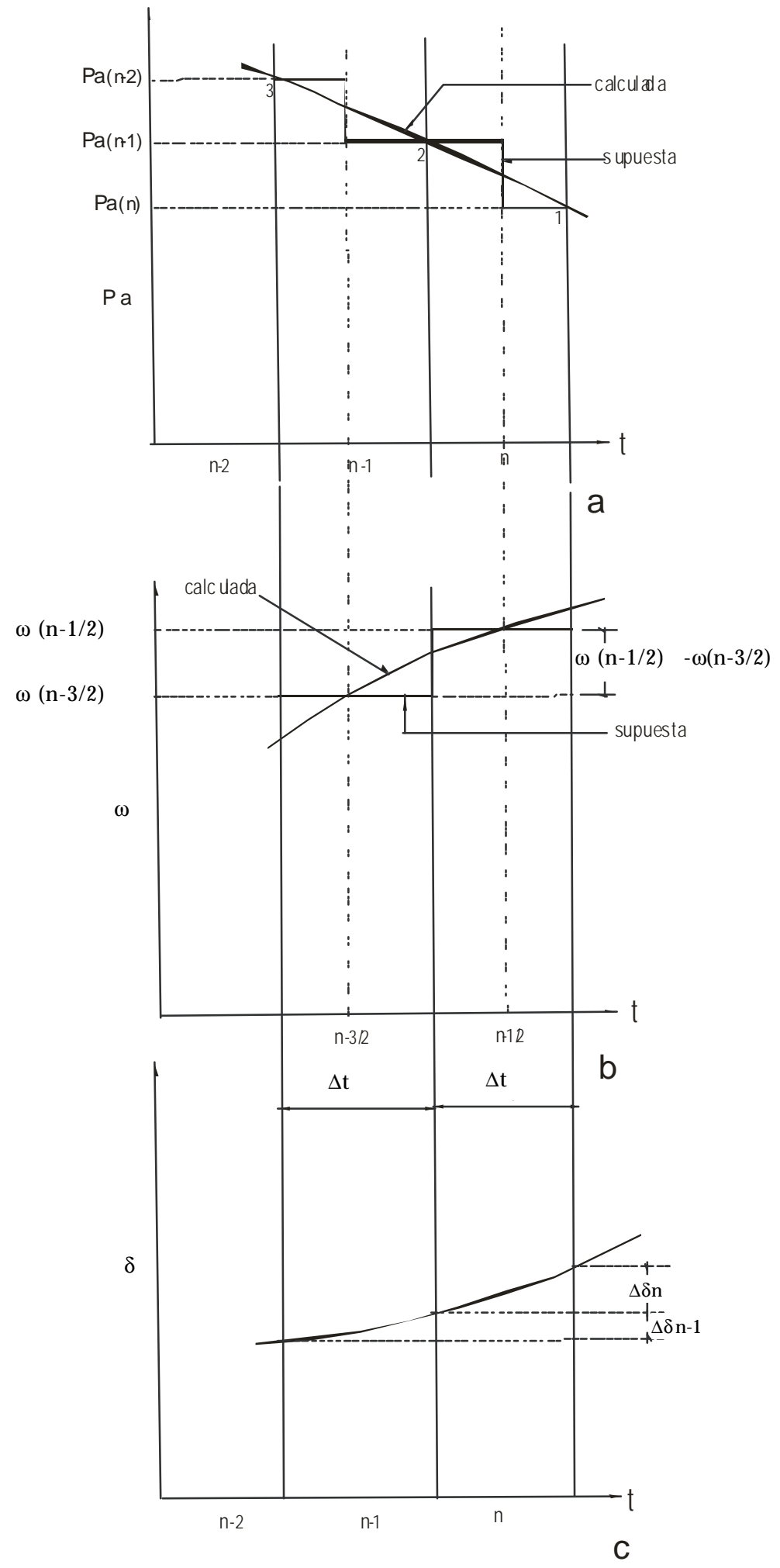

Figura 4. Valores reales y supuestos de de $\omega, \delta$ y $\mathrm{Pa}$ 


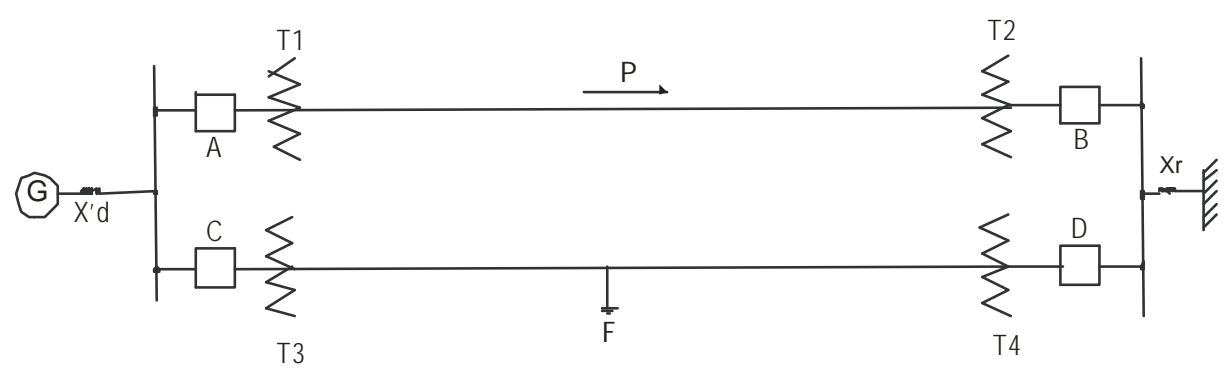

Figura 5. Diagrama unifilar del Sistema Eléctrico de Potencia

Cada una de las líneas tiene un transformador elevador y uno reductor en cada extremo, así como un interruptor que separa a cada línea de la carga y del generador. Los parámetros por conveniencia están dados por unidad, como todos, en base al generador.

\section{Conclusiones}

El problema de estabilidad de un generador síncrono, implica entre varios objetivos, el análisis del comportamiento de los elementos físicos que componen una red eléctrica, como cables que transmiten potencia eléctrica, transformadores de potencia, características de la carga eléctrica, así como la cantidad de potencia transferida del generador a la carga en el instante de la falla. Por otro lado, la observación y determinación del ángulo de potencia que alcanza el rotor, debido a la falla que determina si el generador pierde sincronismo, y por tal razón, se desconecta del sistema. El análisis del deslizamiento del ángulo delta versus tiempo, determina la velocidad de respuesta del equipo de control de la máquina impulsora, así como de los relevadores de protección de los interruptores de las líneas y los del generador. Por último, se genera la intención de dar a conocer un algoritmo a los interesados en estudios de dinámica.

\section{Información del sistema}

El sistema consiste de un generador conectado a una carga por el extremo de dos líneas paralelas. Es decir, las líneas son conectadas en sus extremos por dos barras de donde se unen, por un lado, el generador y por el extremo opuesto una carga que demanda la energía de éste.

Las líneas como protección, cuentan con un interruptor en sus extremos que las separa con sus respectivos transformadores de las barras, en el caso de una falla en ellas.

El generador transfiere, por la diferencia de potenciales entre la barra del transmisor y la barra del receptor, un porcentaje de su potencia, pudiendo ser también el 100\%.

En cierto instante, una de las líneas sufre un desperfecto en el punto $\mathrm{F}$ y durante el corto tiempo que el dispositivo censor instruye al mecanismo de control de la máquina impulsora, tal como compuertas o válvulas, el rotor no tiene freno, que es en sí, la propia carga eléctrica, por lo que se sale de sincronismo.

La falla es de las llamadas sostenidas, de tal manera que la malla eléctrica se ve modificada por la reestructuración que se dio al aislar la falla y la sobrante continúa activa sin aislar al generador. La falla ocurre a la mitad de la línea que une los interruptores C y D.

El problema que se presenta es un ejemplo de muchos, en donde los parámetros son 
Aplicación del algoritmo de solución paso-a-paso de la ecuación que determina la estabilidad ...

tales que el rotor no se desliza, sino que retoma en cierto tiempo su posición original.

El interesado puede variar los parámetros sugeridos en este trabajo y comprobar por su cuenta, con datos propios.

\section{Datos del problema}

S: potencia aparente nominal del generador: $100000000 \mathrm{VA}$

V: tensión nominal del generador:13800 volts.

$X^{\prime} \mathrm{d}$ : reactancia transitoria del generador: $0.476 \mathrm{pu}$

fp: factor de potencia: 0.9

f: frecuencia: 60 Hertz

$\mathrm{H}$ : constante de inercia: 7.0 mega-Jouls MVA

p: potencia transferida: $0.95 \mathrm{pu}$

XL: reactancias de las líneas: $0.3465 \mathrm{pu}$

$X T$ : reactancias de los transformadores: $0.293 \mathrm{pu}$

$\% \mathrm{~V}$ : tensión en por ciento del nodo de la carga: $0.9 \mathrm{pu}$

Xr: reactancia en el nodo de carga: $0.476 \mathrm{pu}$ t1: 1er tiempo de abertura:0.08 seg

t2: $2^{\circ}$ tiempo de abertura: 0.12 seg

tf: tiempo de duración :0.20 seg

\section{Curva de oscilación del rotor de un generador de polos lisos como respuesta a una falla de un sistema eléctrico de potencia}

Con la información solicitada por el programa y ya aplicado "enter" en la pantalla de trabajo, aparecen en el orden siguiente los valores calculados de: Vn, In, Ia, fp, Xe1, Eac, delta y P11, tensión nominal, corriente nominal, corriente de armadura, factor de potencia, reactancia equivalente antes de la falla, tensión inducida, ángulo de fase y potencia eléctrica antes de la falla. Xe2 y P22 reactancia equivalente y potencia eléctrica durante la falla y Xe3 y P33, reactancia y potencia eléctrica después de la falla. Los tiempos de apertura de los interruptores deben ser mayores a 0.01 y además múltiplos a éste. Se estima que el valor final del estudio es suficiente (Gráfica 1).

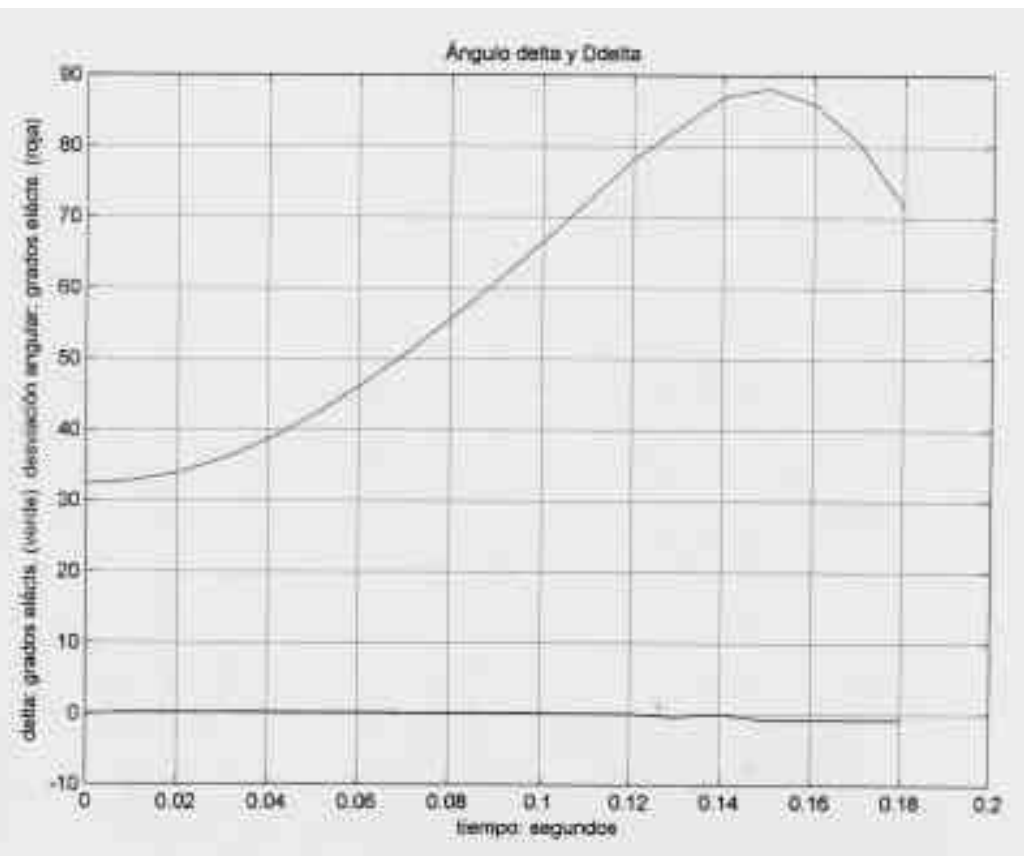

Gráfica 1 
Programa de cómputo

CLF

VA=input ([ 'Pot. aparente

nominal:', num2str(i),'= ']);
V=input (['voltaje
nominal:', num2str(i),'=

Xd=input ([ 'reactancia trans ] );

gen:', num2 str $(1), '=1])$;

fp=input (['factor de potencia

$\because$, num2str(1),'=' ]);

H=input ( [ 'momento

cinetico:', num2str (1),'= ']); $p=i n p u t$ ( [ 'porciento de potencia

transferida:', num2str(1),'= ']);

XL1=input (['reactancia de la linea

']);

$$
1: ', \text { num2atr }(1),{ }^{\prime}=
$$

XI2-input (['reactancia de la linea

$$
2:^{\prime}, \text { num2str (i), }=
$$

']); XT1=input (['reactancia del transfor.

$$
1: ', \text { num2str }\{i\},,^{\prime}=\text { ']); }
$$

XT2=input (['reactancia del transfor.

$2: '$, num2atr $\{i\},{ }^{\prime}=$ ']);

xr3=input (['reactancia del transfor.

$$
3: ', \operatorname{num} 2 \operatorname{str}\{i\},,^{\prime}=\text { ']); }
$$

XT4-input (['reactancia del transfor.

VI-input ( [ $4:$ ', num2str $\{i\},{ }^{\prime}=$ ']); tension del

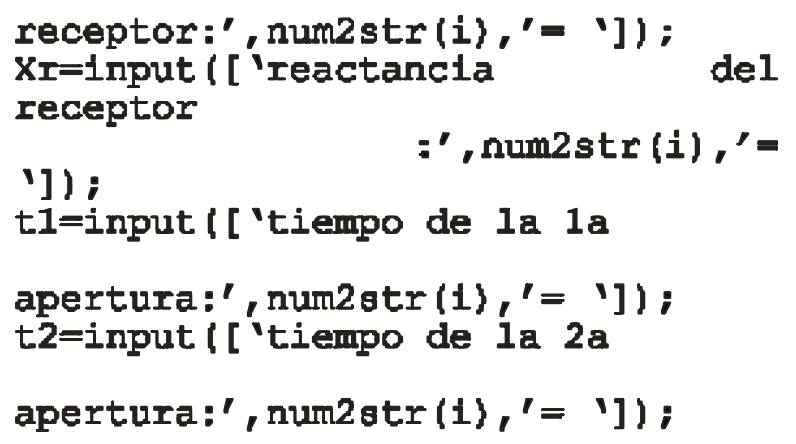

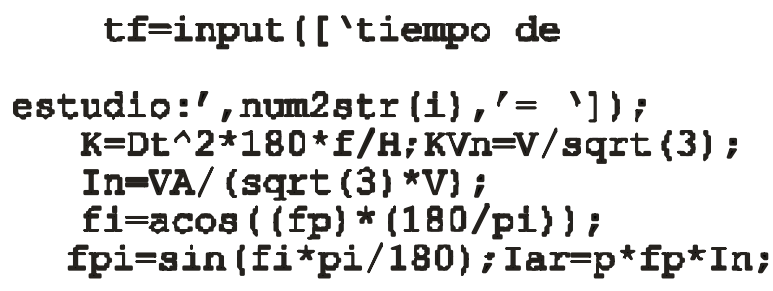

Iai=p*fpi*In; Iar1=Iar^2; Ia11=Ia1^

2;

Ia-sqrt (Iarl+Iail);

\&f $1=\operatorname{atan}(\operatorname{Ia} 1 / \operatorname{Iar}) *(180 / \mathrm{p} 1)$;

$\mathrm{KVb}=\mathrm{Vr} * \mathrm{KVn} ; \mathrm{XB}=\mathrm{KVn} / \mathrm{In}$;

$\mathrm{Xr}=\mathrm{XDr} / \mathrm{XB} ; \mathrm{Xea}=(\mathrm{Xd}+\mathrm{Xr})$;

$\mathrm{Xeb}=(\mathrm{XT} 1+\mathrm{XL} 1+\mathrm{XT} 3)$ * $(\mathrm{XT} 3+\mathrm{XL} 2+\mathrm{XT} 4) /$

$(\mathrm{XT} 1+\mathrm{XL} 1+\mathrm{XT} 2+\mathrm{XT} 3+\mathrm{XL} 2+\mathrm{XT} 4)$;

$\mathrm{Xel}=\mathrm{Xea}+\mathrm{Xeb} ; \mathrm{El}=\mathrm{KVb}+\mathrm{Xel}$ *IaI;

$\mathrm{E} 2=\mathrm{Xe} 1$ * Iar;

$E a c=\operatorname{sqrt}\left((E 1)^{\wedge} 2+(E 2)^{\wedge} 2\right)$ :

delta (1) =atan (E2/E1)*180/p1;

$\mathrm{Pe} 1=3 * \mathrm{KVb}$ * Ia $\mathrm{fp} / \mathrm{VA}$;

$\mathrm{Rx} 1=\mathrm{XT} 1+\mathrm{XL} 1+\mathrm{XT} 2$;

$\mathrm{Rx} 2=\mathrm{XI} 2 / 2+\mathrm{XT} 4$;

$\mathrm{Rx} 3=\mathrm{XT} 3+\mathrm{XL} 2 / 2$;

$\mathrm{R} 4=\mathrm{R} \times 1+\mathrm{R} \times 2+\mathrm{R} \times 3$;

$\mathrm{Ra}=\mathrm{R} \times 3 * \mathrm{R} \times 2 / \mathrm{R} 4$;

$\mathrm{Rb}=\mathrm{R} \times 3 * \mathrm{R} \times 1 / \mathrm{R} 4$;

$\mathrm{Rc}=\mathrm{R} \times 1 * \mathrm{R} \times 2 / \mathrm{R} 4$;

$\mathrm{RI}=\mathrm{Xr}+\mathrm{Rc} ; \mathrm{Rg}=\mathrm{Xd}+\mathrm{Rb}$;

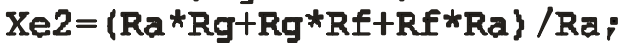

P22 $=3 * \mathrm{Eac}^{\star} \mathrm{KVb} /\left(\mathrm{Xe}{ }^{\star}{ }^{\mathrm{VAA}}\right)$;

$\mathrm{Xe3}=\mathrm{Xd}+\mathrm{RxI}+\mathrm{Xr}$;

$\mathrm{P33}=3 * \mathrm{Eac} * \mathrm{KVb} /(\mathrm{Xe} 3 * \mathrm{VA})$;

$\mathrm{P} 11=\mathrm{Pe} 1 ; \mathrm{Dt}=0.01$;

$t(1)=0 ; n=1 ;$

Pm=P11*sin (delta*pi/180) ;

$\operatorname{Pe}(a, n)=P 22 ; n=n+1$;

$P(;, n)=P 22 * \sin (\operatorname{delta}(1) * \mathrm{p} 1 / 180)$;

$\operatorname{Pe}(a, n)=\operatorname{Pm}+\mathrm{P}(a, n) / 2$;

$\operatorname{Pa}(a, n)=\operatorname{Pm}-\operatorname{Pe}(:, n)$;

$\operatorname{KPa}(:, n)=\mathbb{R} * \mathrm{~Pa}(:, n) ;$

Ddelta $(:, n)=\operatorname{KPa}(:, n)$;

delta $(:, n)=\operatorname{delta}(1) ; t(n)=0.0001$;

while $t<t l n=n+1$;

$\operatorname{Pe}(:, \pi)=P 22 * a 1 n($ delta $\{:, n-$

$\operatorname{Pa}(:, n)=\operatorname{Pm}-\operatorname{Pe}(:, n)$;

1) $* 3.1415937 / 180$ ):

$\operatorname{KPa}(:, \Pi)=K * \operatorname{Pa}(:, \Pi) ;$

Ddelta $(:, \Omega)=\operatorname{Ddelta}(:, n-$

1) $+\operatorname{KPa}(:, n)$;

$\operatorname{delta}(:, n)=\operatorname{delta}(:, n-$

1) +Ddelta $(:, n)$;

$t(n)=(n-2) * D t ;$ end

$\mathrm{n}=\mathrm{n}+1$ 


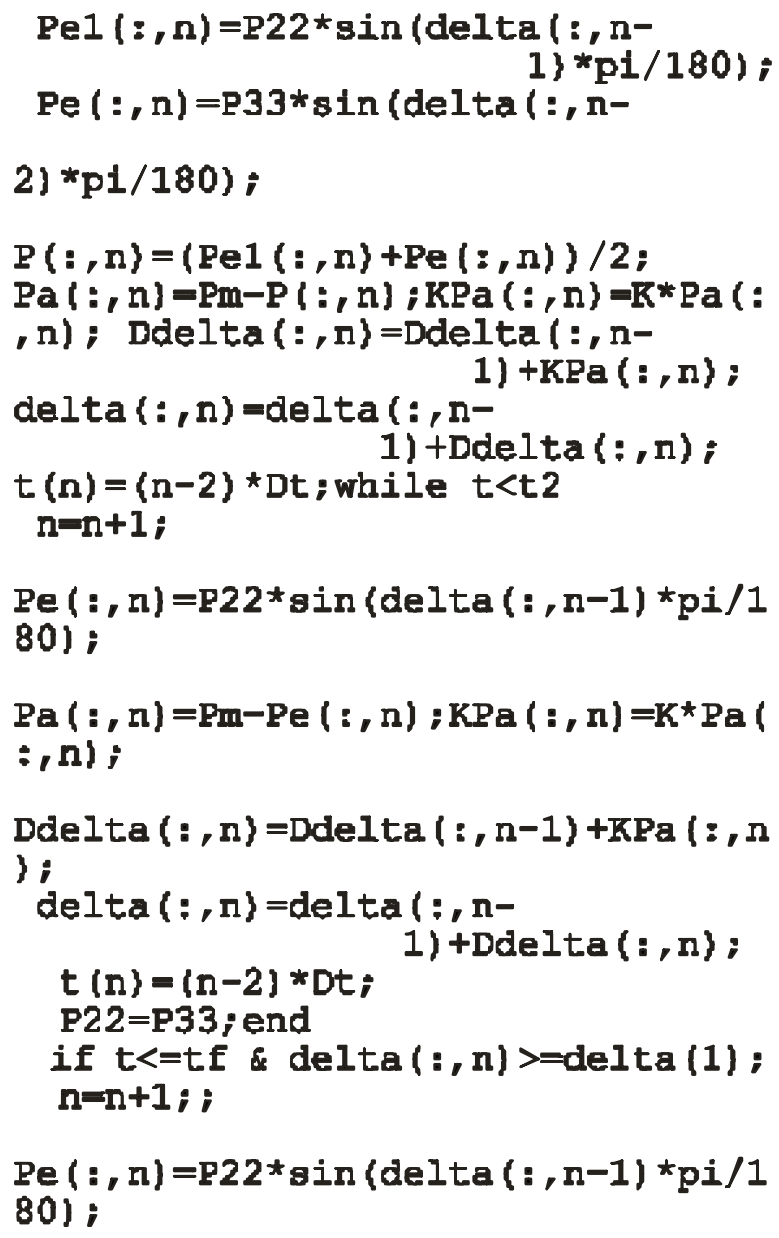

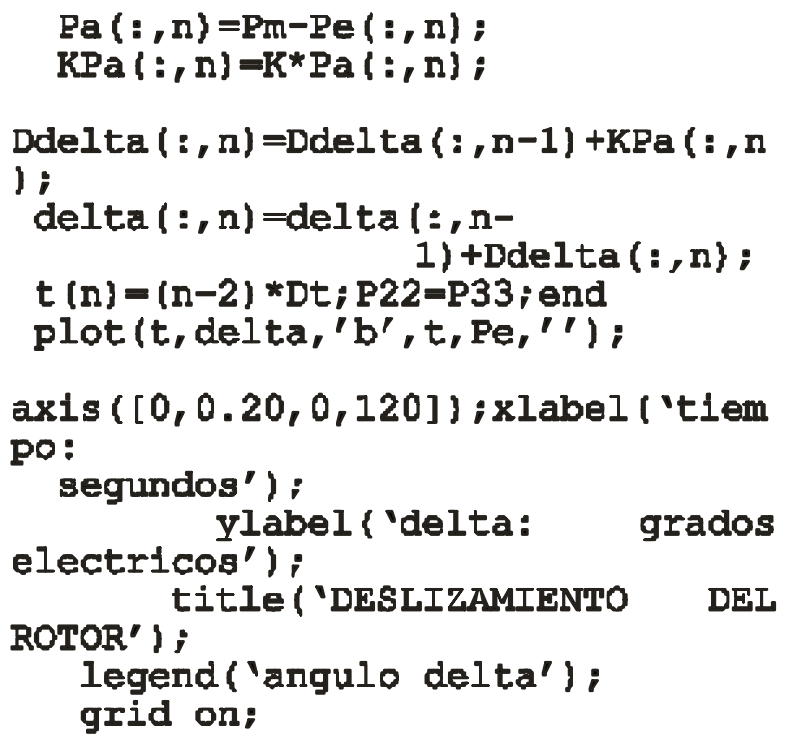

\section{Referencias}

Luthe A., Olivera A., Schutz F. (1985). Métodos numéricos. Limusa, México, pp. 215-220.

Stevenson W.D. Jr. (1982). Elements of Power System Analysis. International Student Edition, México, pp. 409-416.

Enriquez H.G.(1982). Sistemas eléctricos de potencia. Limusa, México, pp. 184-191.

\section{Semblanza del autor}

Hugo Alfredo Grajales-Román. Obtuvo la licenciatura en ingeniería mecánica eléctrica en la Facultad de Ingeniería de la UNAM. Realizó estudios de posgrado en la Universidad de Houston Texas, EE.UU. y en la entonces División de Estudios Superiores de la FI, UNAM. En México, dentro de la Comisión Federal de Electricidad laboró como ingeniero de puestas en servicio de plantas, así como asesor en la adquisición de equipo para plantas. Fue investigador comisionado en el Instituto de Ingeniería, UNAM con la realización de un proyecto para Centro Nacional de Control de Energía, CENACE. Fue gerente de operación y planeación de plantas en Tabacos Mexicanos. Ha sido profesor durante 31 años en la Facultad de Ingeniería, UNAM. 